Revue pluridisciplinaire du monde antique

19 | 2003

Le statut et l'image du corps dans la mythologie et la littérature grecques (suite et fin)

\title{
Corps et mémoire à Rome
}

\section{Catherine Baroin}

\section{OpenEdition}

\section{Journals}

Édition électronique

URL : https://journals.openedition.org/kentron/1859

DOI : 10.4000/kentron. 1859

ISSN : 2264-1459

\section{Éditeur}

Presses universitaires de Caen

\section{Édition imprimée}

Date de publication : 31 décembre 2003

Pagination : 159-178

ISBN : 2-84133-222-5

ISSN : 0765-0590

\section{Référence électronique}

Catherine Baroin, "Corps et mémoire à Rome », Kentron [En ligne], 19 | 2003, mis en ligne le 12 avril 2018, consulté le 13 octobre 2022. URL : http://journals.openedition.org/kentron/1859 ; DOI : https:// doi.org/10.4000/kentron.1859

\section{(c) (i) (9)}

Creative Commons - Attribution - Pas d'Utilisation Commerciale - Pas de Modification 4.0 International - CC BY-NC-ND 4.0

https://creativecommons.org/licenses/by-nc-nd/4.0/ 


\section{CORPS ET MÉMOIRE À ROME}

D’après l'ensemble des textes latins datant du dernier siècle de la République et du Haut-Empire, il n'y a pas, à Rome, une représentation cohérente et unifiée de la mémoire - pourquoi y en aurait-il une, d'ailleurs ${ }^{1}$ ? - , représentation qui la rattacherait de façon systématique à l'âme (animus) ${ }^{2}$ ou à l'esprit (mens) ou au corps (corpus) ou aux sens (sensus), etc.

À ce titre, le lexique est révélateur ${ }^{3}$. Le vocabulaire latin de la mémoire est majoritairement dérivé de la racine indo-européenne *men-, qui indique une activité intérieure; il en va ainsi des termes memini, memoria, mais aussi moneo, monumen-

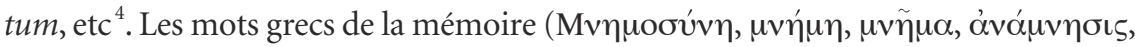
$\mu ц \nu \eta \dot{\sigma \kappa о \mu \alpha \iota, ~ e t c .) ~ o n t ~ l a ~ m e ̂ m e ~ o r i g i n e ~}{ }^{5}$. Cependant existent aussi en latin les termes recordatio et recordor, formés sur le mot cor $(\text { cœur })^{6}$. Le verbe recordor est parfois utilisé pour désigner précisément le fait de retrouver un souvenir et, dans ce cas, le contexte explicite cette signification ${ }^{7}$. Toutefois, le lexique latin ne distingue pas,

1. Pour notre époque, voir Le Breton 1985, 193-200 : s'il y a sans doute dans l'Occident moderne un "modèle hégémonique du corps [...] constitué par le savoir anatomo-physiologique» (p. 193), le corps n'en est pas moins l'objet de multiples représentations, qui n'ont pas entre elles de cohérence et ne visent pas à en avoir.

2. Nous traduisons animus par âme, sachant bien que cette traduction est imparfaite, puisque le mot latin a des significations différentes (intention, sentiments, courage, etc.) selon les contextes et que le terme français est chargé de connotations qui n'existent pas en latin pour la période qui nous intéresse.

3. Cet article reprend des éléments développés dans ma thèse de doctorat (Baroin 1998).

4. Voir Ernout \& Meillet 1967, 395, s.v. memini (la racine * men- « indiquait les mouvements de l'esprit»). Voir aussi Simondon 1982, 19: «La notion primitive impliquée dans la racine [est] celle d'une activité, d'une énergie mentale qui s'exerce dans des opérations comme penser, faire attention à, tourner son esprit vers, se rappeler. » Le mot latin mens (dont l'une des traductions usuelles est « esprit ») est aussi formé sur cette racine (voir Ernout \& Meillet 1967, 396, s.v. mens). Toutefois, cette étymologie ne signifie nullement que, pour les Romains, la mémoire soit purement et uniquement une faculté mentale.

5. Pour les mots grecs formés sur cette racine, voir Chantraine 1968-1975; Ernout \& Meillet 1967, 395; Simondon 1982, 19.

6. Voir Varron, De lingua Latina, VI, 46: «Recordari (se rappeler), c'est rappeler dans son cœur (Recordari rursus in cor revocare). » Voir aussi Ernout \& Meillet 1967, 142, s.v. cor.

7. Cicéron (Tusculanes, I, 57-58) utilise recordatio (qui traduit óvóuvๆoı૬) et recordari ou reminiscor pour rendre compte de la théorie platonicienne de l'anamnèse. Ces deux verbes sont employés dans le même contexte dans le Cato Maior, 78. Sur l'emploi de memoria et recordatio chez Cicéron pour 
la plupart du temps, entre mémoire et remémoration ${ }^{8}$. Ce n'est donc pas à une telle distinction que renvoie la double étymologie du vocabulaire latin de la mémoire. Elle ne définit pas non plus deux mémoires « différentes », l'une qui serait rattachée à l'esprit, et l'autre au cœur ${ }^{9}$. En fait, la différence entre memini et recordor tient essentiellement à leur fréquence dans la langue écrite: recordor et recordatio se rencontrent beaucoup moins souvent que memini, memoria et leurs composés ${ }^{10}$.

D'autre part, afin de s'interroger sur les rapports entre la mémoire et le corps dans une perspective anthropologique, il faut prendre en compte des documents de genres très divers sans les hiérarchiser : on ne peut considérer, par exemple, que les textes médicaux donneraient accès à une « vérité» de la question, davantage que des textes dits «littéraires». Autrement dit, il n’y a pas, d'un côté, des documents qui montreraient un corps réel et, de l'autre, ceux qui dessineraient un corps imaginaire. Les deux dimensions sont indissociables ${ }^{11}$.

Si on l'étudie dans une telle perspective, la mémoire apparait comme une caractéristique identitaire de l'homme romain (tel qu'il apparait dans le corpus que nous avons défini ${ }^{12}$ ); elle joue un rôle fondamental dans sa relation à lui-même ${ }^{13}$ et aux autres. En même temps, elle est plus spécialement liée à certaines parties du corps. Elle n'en est pas pour autant localisée dans le corps sur le plan anatomique; en effet, cette relation avec des parties du corps révèle elle aussi l'importance de la mémoire

rendre compte de ce qu'est la mémoire selon les Épicuriens, voir le De finibus, II, 96-106, et les commentaires de Pigeaud 1981, 166-169. Pour des emplois marqués de recordor et recordatio, en dehors de toute référence à Platon, cf. Cicéron, Pro Sestio, 11 ; Pline le Jeune, Lettres, I, 9, 3.

8. C'est pour cette raison que, dans cette étude, nous ne distinguerons pas non plus entre les deux. En revanche, la distinction est exprimée en grec par les termes $\mu v \eta \dot{\mu \eta}$ et óvó $\mu v \eta \sigma \iota \varsigma$. Pour l'explication de ces termes, cf. Simondon 1982, 149, 157-159, 179, 312-315, 322 en particulier.

9. Une telle conception repose en partie sur un a priori moderne qui différencie l'esprit et le cour. Quant à la locution "par cœur ", apparue vers 1200, elle est, d'après le Dictionnaire historique de la langue française (A. Rey (dir.), Paris, Le Robert, 1992, s.v. cœur), " le vestige du sens large ancien "siège de la mémoire" ». À la Renaissance, le mot cœur peut signifier «mémoire», mais la locution "par cœur » veut dire «dans l'imagination, en apparence» (A.J. Greimas et T.M. Keane, Dictionnaire du moyen français, La Renaissance, Paris, Larousse, 1992, s.v. cueur, cuer). Aujourd'hui, lorsque nous utilisons l'expression "par cœur », qui renvoie à la mémoire, nous n’attribuons pas de rôle spécifique au cour comme organe.

10. Par exemple, si l'on envisage un corpus homogène, on compte 93 formes de memini et 34 de recordor dans la correspondance de Cicéron; chez Tacite, le rapport est analogue : il y a 26 occurrences du verbe memini pour 5 de recordor (dont deux participes passés).

11. On peut citer ici une formule de D. Le Breton $(1985,194)$ : «Comme tout ce qui relève du sémantique, le corps est une fiction bien réelle [...].»

12. Il s'agit donc essentiellement des hommes de l'élite politique et sociale de l'Urbs, appartenant à la minorité cultivée.

13. Nous pensons ici à la mémoire et à l'oubli de soi, exprimés par les locutions meminisse sui ou memor sui, et leurs contraires obliuisci sui, oblitus esse sui ou obliuio sui. Sur cet aspect, voir Baroin 1998, $350 \mathrm{sq}$. 
dans les relations interpersonnelles et elle permet de comprendre comment est construit un corps culturel.

\section{L'homme, "animal memor»}

À Rome, le fait d'avoir de la mémoire (memoria) ou d'être memor est une caractéristique identitaire ${ }^{14}$, au point que, dans le De legibus, Cicéron définit l'homme (homo) dans les termes suivants :

[...] cet être vivant qui est prévoyant, pénétrant, multiple, qui a de l'acuité, de la mémoire $\left[\right.$ memor], qui est plein de raison et d'intelligence, que nous appelons homme ${ }^{15}$.

Cette définition de l'homme est philosophique, mais elle a en même temps une valeur anthropologique. On la retrouve par exemple dans le contexte oratoire. Ainsi, selon Cicéron, la mémoire fait partie des caractéristiques de la nature (natura) de l'homme qu'un avocat doit envisager dans sa confirmation (confirmatio). Au même titre que le sexe (sexus), le peuple (natio), la patrie (patria), la parenté (cognatio) et l'âge (aetas), l'avocat prend en compte « les qualités ou les défauts qui ont été donnés par la nature à l'âme [animus] ou au corps [corpus] », c'est-à-dire le fait qu'un être humain soit

fort ou faible, grand ou petit, beau ou laid, rapide ou lent, aigu ou particulièrement émoussé, doué de mémoire ou oublieux [memor an obliuiosus], aimable ou grossier, sachant se tenir ou non ${ }^{16}$.

Cicéron énumère d'abord, ici, des caractéristiques physiques, puis intellectuelles et morales à la fois, sans que le partage entre attributs de l'âme et attributs du corps soit toujours clairement établi - qu'en est-il de la rapidité et de la lenteur ?... Il semble cependant que le fait d'avoir ou non de la mémoire soit ici à mettre du

14. Notons qu'il existe un cognomen Memor, dont le sens littéral renvoie à une qualité de la personne (comme les cognomina Castus, Clemens, etc., qui sont très fréquents : voir Kajanto 1982, 68). Cependant, le fait que ce cognomen existe (il est d'ailleurs rare: voir Kajanto 1982, 251) ne prouve rien quant à l'importance, dans la culture romaine, de la mémoire comme valeur : que faudrait-il dire, alors, de Cicero?

15. Cicéron, De legibus, I, 22: « [...] animal hoc prouidum, sagax, multiplex, acutum, memor, plenum rationis et consilii, quem uocamus hominem » (toutes les traductions de textes anciens sont personnelles, sauf indication contraire). C'est Cicéron qui parle ici; il explique que la raison (ratio) existe dans l'homme et dans la divinité.

16. Cicéron, De inventione, I, 35: «Praeterea commoda et incommoda considerantur ab natura data animo aut corpori, hoc modo: ualens an imbecillus, longus an breuis, formosus an deformis, uelox an tardus sit, acutus an hebetior, memor an obliuiosus, comis an infacetus, pudens, patiens an contra. " Il s'agit bien des dispositions naturelles de chacun, non de ce qui constitue une manière d'être ( $h a-$ bitus), acquise par le travail (industria) et l'application (studium) : ibid., 35-36. 
côté de l'animus, par distinction avec le corpus. Dans les traités oratoires ${ }^{17}$ et dans les textes philosophiques de Cicéron, la mémoire est de façon générale rattachée à l'âme (animus), dont elle est un des attributs. Ainsi, dans un passage du premier livre des Tusculanes (54-66), Cicéron s'interroge longuement sur la mémoire (memoria) et sur l'âme (animus). Il rappelle la théorie de la réminiscence platonicienne ${ }^{18}$, il s'oppose au point de vue des Épicuriens, qui rattachent la mémoire au corps et aux atomes $^{19}$, et il propose aussi deux images, dont celle de la tablette de cire, pour tenter de décrire la mémoire, tout en montrant qu'elles sont inadéquates ${ }^{20}$. S'il attribue la mémoire à l'âme (animus), qui est de nature divine ${ }^{21}$, il ne donne volontairement pas de définition, du moins pas de définition unique de la mémoire. Cependant, si Cicéron rejette dans ce passage la comparaison avec la tablette de cire, il y recourt dans des traités oratoires pour expliquer ce que sont les lieux et les images de l'art de la mémoire (ars memoriae) ${ }^{22}$. Cette représentation est donc bien pertinente dans la culture romaine.

On voit qu'il n'y a pas un discours unique sur la mémoire, mais des discours. Ainsi, selon l'auteur et surtout le genre, le contexte et la stratégie du texte, la mémoire peut être considérée comme une partie ou un attribut de l'âme (animus), ou bien être en relation avec l'esprit (mens ${ }^{23}$; l'ingenium ${ }^{24}$ (l'intelligence, le tempérament) peut être qualifié d' « oublieux »; il en va de même pour le cœur $(\text { cor })^{25}$. La mémoire

17. Voir encore Cicéron, De inventione, II, 160 : «La mémoire est ce par quoi l'âme retrouve les choses qui sont passées " (Memoria est per quam animus repetit illa quae fuerunt).

18. Cicéron, Tusculanes, I, 57-58: voir supra note 7.

19. Ibid., 60: «Elle [la mémoire] ne relève certainement pas du cœur, ni du sang, ni du cerveau, ni des atomes; est-ce du souffle, du feu ? Je l'ignore» (Non est certe nec cordis nec sanguinis nec cerebri nec atomorum; animae sit ignisne nescio).

20. Ibid., 61: pour comprendre ce qu'est la mémoire, Cicéron propose deux hypothèses et les repousse aussitôt : on ne peut dire qu'il y ait dans l'âme (animus) un « réservoir » (capacitas); on ne peut dire non plus que l'âme soit comme une tablette de cire (cera) et que la mémoire soit constituée d'empreintes (uestigia) des choses dans l'esprit (mens).

21. Ibid., 57: «Habet primum memoriam [animus].» Il y a dans l'âme [animus] des éléments divins (ibid., 56 : «inesse in animis hominum diuina quaedam»). Voir aussi I, 60 et 65-66 (65: l'âme est divine: «Ergo animus $q<$ uoque $>$, ut ego dico, diuinus est [...]»). Voir enfin Cato Maior, 78 : la mémoire du passé (memoria praeteritorum) est l'une des preuves de l'immortalité de l'âme.

22. Cicéron, De oratore, II, 354; Partitiones oratoriae, 2, 3 et 26. Voir aussi Ad Herennium, III, 30 ; Quintilien, Institution oratoire, XI, 2, 21.

23. Voir Cicéron, Tusculanes, 1, 61 (voir supra note 20 : la mens est en jeu); Catulle, Poésies, 64, 248 : le mot mens est qualifié par l'adjectif «oublieux» (immemor). Voir aussi Ovide, Pontiques, III, 5, 41: " mens oblita quid absit. » Aux VI-VII siècles, Isidore de Séville (Étymogies, XI, 1, 13) identifie memoria et mens («[...] memoria mens est, unde et immemores amentes»).

24. Cicéron, Brutus, 218 : l'ingenium est qualifié par l'adjectif immemor. Voir aussi Quintilien, Institution oratoire, I, 3, 1 ( Ingenii signum in paruis praecipuum memoria est»).

25. Cf. Virgile, Énéide, IX, 225 (corda oblita laborum); Catulle, Poésies, 64, 231 (memori... corde) : voir infra note 66. 
peut encore être mise en relation avec les sens (sensus) ${ }^{26}$. Par ailleurs, la mémoire est associée à des parties du corps; nous y reviendrons.

En outre, on peut trouver à l'intérieur d'un même texte, des assertions qui paraissent contradictoires. Ainsi, d'après Sénèque le rhéteur, si la mémoire est bien une partie de l'animus, elle est nommée à la suite de qualités des organes de la sensibilité :

Mais bien que la vieillesse me donne à regretter déjà plusieurs de mes avantages, qu'elle ait émoussé l'acuité de mes yeux, diminué la finesse de mon ouïe, et affaibli la solidité de mes nerfs, plus que tout ce dont je viens de parler, la mémoire, la partie la plus sensible et la plus fragile de l'âme, est celle à laquelle la vieillesse s'attaque d'abord ${ }^{27}$.

On voit bien qu'ici il n’y a pas de séparation tranchée entre des qualités physiques et ce qui relève de l'animus. En outre, ce passage met l'accent sur une dimension fonctionnelle de la mémoire, dimension essentielle pour comprendre les discours latins sur la memoria. Enfin, il indique que la mémoire diminue avec l'âge. Ainsi, un peu plus loin, Sénèque se plaint de ne plus contrôler sa mémoire, qui désormais lui obéit mal (precario paret) ${ }^{28}$. En fait, la mémoire de Sénèque vieillissant se comporte comme un mauvais esclave, absent quand on a besoin de lui, loyal autrefois et désormais infidèle. L'idée que la mémoire est transformée et altérée par le vieillissement est solidaire de la représentation générale du senex, dont le corps est affaibli et dont l'activité politique et sociale a diminué. Cette conception montre bien que la mémoire est liée au corps (corpus) et à l'état général (habitus).

Dans le De senectute, Cicéron met dans la bouche de Caton l'Ancien l'opinion selon laquelle la mémoire diminue avec l'âge ; mais Caton ne l'exprime que pour la réfuter. En effet, lui-même, dit-il, a une excellente mémoire ${ }^{29}$; pour les autres, il précise :

Je n'ai jamais entendu dire qu'un vieillard avait oublié l'endroit où il avait enfoui son trésor ; les vieillards se souviennent des affaires dont ils s'occupent, des engagements pris sous caution, de leurs débiteurs et de leurs créanciers ${ }^{30}$.

26. Voir Horace, Épodes, 14, 1-2 (cité infra note 48).

27. Sénèque le rhéteur, Controverses, I, préface $2:$ :Sed cum multa iam mihi ex meis desideranda senectus fecerit, oculorum aciem retuderit, aurium sensum hebetauerit, neruorum firmitatem fatigauerit, ante ea quae rettuli, memoria est res ex omnibus animi partibus maxime delicata et fragilis, in quam primam senectus incurrit. "

28. Ibid., préface 5 .

29. Cicéron, Cato Maior, 21.

30. Ibid.: "Nec uero quemquam senem audiui oblitum quo loco thesaurum obruisset; omnia quae curant meminerunt, uadimonia constituta, qui sibi, cui ipsi debeant. " Le trésor est l'une des métaphores qui, dans les textes latins, désignent la mémoire elle-même (voir par exemple Ad Herennium, III, 28 ; Quintilien, Institution oratoire, XI, 2, 1, etc.). Il y a peut-être ici un jeu avec cette image bien 
Le type d'activités décrit ici par Caton correspond à la gestion d'un patrimoine et de ressources financières: même si les vieillards ne participent plus à la vie politique ${ }^{31}$, ils doivent encore gérer leurs biens, ce qui entretient une mémoire relationnelle. La perte de cette mémoire relationnelle est du reste un des symptômes traditionnels du vieillissement ${ }^{32}$. Selon Caton, les vieillards qui courent le risque de perdre la mémoire sont ceux qui sont par nature plus lents que les autres, ceux qui n'exercent pas leur mémoire ${ }^{33}$, ceux dont la vieillesse est «inactive, paresseuse, somnolente », à l'instar des senes de la comédie latine, qui sont « crédules, oublieux [obliuiosi], ramollis » ${ }^{34}$. Caton recommande de lutter (pugnare) contre les effets du vieillissement en exerçant sa mémoire, mais aussi en respectant la frugalité et la sobriété, afin de refaire (reficere) ses forces sans les écraser (opprimere), en veillant tant au bon état du corps (corpus) qu'à celui de l'esprit (mens) et de l'âme (animus) ${ }^{35}$.

On comprend que le vieillissement du corps et de la mémoire est autant culturel que biologique : les vieillards « oublieux» ne font pas partie de l'élite (à laquelle appartient Caton), ils font partie du monde de la comédie ou de la satire. D’autre part, l'hygiène de vie préconisée par Caton est à la fois physique, intellectuelle et morale. Dans le Cato Maior, elle est recommandée aux vieillards, mais elle est, en fait, un prolongement des règles que doit suivre un homme en âge de faire une carrière

connue. On peut y voir aussi une allusion à certains vieillards de comédie (l'Euclion de La Marmite, par exemple), évoqués explicitement plus loin.

31. Cicéron, Cato Maior, 35.

32. Cf. Juvénal, Satires, X, 232-237 : «Chose pire encore que toutes les déchéances physiques, le vieillard est fou : il ne connait plus le nom de ses esclaves / ni le visage d'un ami (omni / membrorum damno maior dementia, quae nec / nomina seruorum nec uultum agnoscit amici) avec lequel il a dîné la nuit passée, ni les enfants qu'il a engendrés, qu'il a élevés. » Voir aussi Satiricon, 117, 10 : Eumolpe feint de ne plus reconnaître ceux qui l'entourent.

33. Cicéron, Cato Maior, 21 : «At memoria minuitur. - Credo, nisi eam exerceas, aut etiam si sis natura tardior. » Pour ces exercices qui concernent spécifiquement la mémoire, voir ibid., 38 : Caton écrit ses Origines; il s'occupe de droit et de littérature grecque; il précise aussi : «à la manière des Pythagoriciens, pour exercer ma mémoire, le soir, je me remets en mémoire ce que j'ai dit, entendu ou fait chaque jour» ([...] Pythagoreorumque more, exercendae memoriae gratia, quid quoque die dixerim,

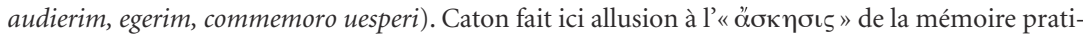
quée par les Pythagoriciens chaque matin et chaque soir. Cet exercice de mémoire visait à acquérir le

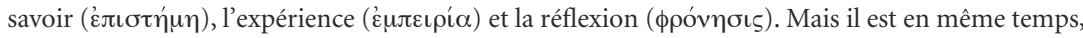
au moins d'après certaines sources (notamment Jamblique, Vers dorés, 40-44), un examen moral, qui tend à la connaissance de soi. Sur tout cela, voir Simondon 1982, 158-160.

34. Ibid., 36: "Nam quos ait Caecilius "comicos stultos senes", hos significat credulos, obliuiosos, dissolutos, quae uitia sunt non senectutis, sed inertis, ignauae, somniculosae senectutis. » La citation provient d'une comédie perdue de Cécilius.

35. Ibid. : «[...] utendum exercitationibus modicis; tantum cibi et potionis adhibendum ut reficiantur uires, non opprimantur. Nec uero corpori solum subueniendum est, sed menti atque animo multo magis [...] ; et corpora quidem exercitationum defatigatione ingrauescunt, animi autem se exercendo leuantur.» 
politique (un iuuenis), s'il veut être un bon orateur ; il en va de même pour l'entraînement (exercitatio) de la mémoire; nous y reviendrons.

La mémoire se trouve donc liée au corps social ${ }^{36}$ autant qu'à un certain état du corps physique (habitus, ualetudo). De ce double aspect témoigne la notice que Pline l'Ancien consacre à la mémoire et à ses dysfonctionnements, notice dans laquelle on retrouve l'idée de fragilité exprimée par Sénèque le rhéteur:

Rien n'est aussi fragile en l'homme [que la mémoire] : les maladies, une chute, et même la peur peuvent lui porter atteinte, soit partiellement, soit totalement. Frappé par une pierre, un homme oublia seulement les lettres; tombé du haut d'un toit très élevé, un autre ne reconnut plus ni sa mère, ni ses parents par alliance, ni ses proches; un autre ne reconnut plus ses esclaves pendant une maladie; l'orateur Messala Corvinus oublia jusqu'à son propre nom. Ainsi, la mémoire essaie et entreprend de nous faire défaut, même quand notre corps est exempt de troubles et en bonne santée ${ }^{37}$.

Les amnésies dont Pline rend compte ici sont essentiellement des privations de la mémoire relationnelle, qui neutralisent le rapport à soi et aux autres, comme on l'a vu dans le Cato Maior. Un homme qui ne reconnaît plus ses proches, qui ne peut répondre à son propre nom est mis en dehors des échanges sociaux, exclu de la pratique de la salutatio, par exemple. En outre, si Pline souligne la relation entre le fonctionnement de la mémoire, d'une part, et, d'autre part, la santé, à la fois physique (morborum iniuriae, quietum corpus et ualidum) et, en quelque sorte, morale (etiam metus sentit), aucune des amnésies qu'il mentionne n'est rattachée à une pathologie précise ni au mauvais fonctionnement d'un organe: ces exemples montrent surtout que la perte totale ou partielle de la mémoire provoque un dérèglement profond de l'individu social.

De même, dans les textes médicaux, la perte, plus ou moins durable, de la mémoire n'est pas envisagée comme une pathologie en soi : elle est un signe de mala$\operatorname{die}^{38}$, par exemple de paralysie nerveuse ${ }^{39}$. À l'inverse, le bon fonctionnement de la

36. J'entends par là le corps de l'homme en tant qu'il est acteur de la vie sociale et de rituels sociaux. En même temps, ces rituels sociaux construisent le corps.

37. Pline l'Ancien, Histoire naturelle, VII, $90:$ :Nec aliud est aeque fragile in homine: morborum et casus iniurias atque etiam metus sentit, alias particulatim, alias uniuersa. Ictus lapide oblitus est litteras tantum; ex praealto tecto lapsus matris et adfinium propinquorumque cepit obliuionem, alius aegrotus seruorum, etiam sui uero nominis Messala Coruinus orator. Itaque saepe deficere temptat ac meditatur uel quieto corpore et ualido. » Ce texte est repris par Solin, I, 110-111.

38. Sur l'oubli de soi, l'oubli des fonctions naturelles et la perte de la mémoire comme signes de maladie chez Hippocrate, voir Pigeaud 1981, 462 (qui renvoie à Épidémies, III, 6 et 17) et 61 (pour Galien). Chez Galien, voir Art médical, XXI, 12 (un oubli inhabituel peut être un signe avant-coureur de maladie). Je n'ai rien trouvé qui concerne la mémoire ou l'oubli chez Scribonius Largus.

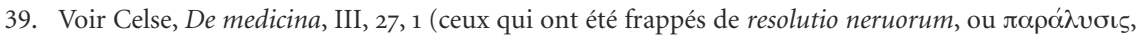
ont perdu la mémoire). 
mémoire est un signe de santé et entraîner sa mémoire peut même avoir une valeur curative $^{40}$. Dans les deux cas, la présence et l'activité de la mémoire sont associées à la santé, santé du corps et de l'âme ou de l'esprit à la fois. En revanche, les textes des médecins ne consacrent pas de développement particulier à des maladies de la mémoire ou à la mémoire en soi $^{41}$; si l'on est tenté d'y chercher quelque chose de tel, c'est par un a priori moderne. En effet, ce sont les affections de la personne et leur traitement qui importent dans le discours médical, à Rome du moins ${ }^{42}$. Mais la mémoire n'y est pas isolée comme si elle était une faculté à étudier pour elle-même, indépendamment du corpus et de l'animus. Il faut donc prendre en compte le discours médical lorsqu'on s'interroge sur le corps et la mémoire, mais il n'est ni plus vrai ni plus riche d'enseignements sur cette question que les textes de Pline l'Ancien ou ceux qui sont issus de traités oratoires.

D'autre part, c'est encore le corps social de l'individu qui est en jeu dans des contextes et des pratiques où il y a perte de mémoire, perte qui n'est pas nécessairement connotée de manière négative. En effet, il existe ce qu'on peut appeler des «nourritures d'oubli » ${ }^{43}$, c'est-à-dire des substances, associées à des comportements,

40. Voir Celse, De medicina, III, 18, 11 et 21: parmi les moyens de guérir la forme aiguë de l'insania, ou $\phi \rho \varepsilon ́ v \eta \sigma \iota \varsigma$, Celse recommande de faire réciter aux malades, s'ils sont lettrés (studiosi litterarum), ce dont ils peuvent se souvenir ( recitare, si qua meminerunt, cogendi sunt»), et encore de les obliger à apprendre quelque chose par cœur et à s'en souvenir ( "ediscere aliquid et meminisse»). Sur la phrénitis (maladie aiguë), voir Pigeaud 1981, 61, 71-100, 347 en particulier.

41. Hippocrate et Galien associent la mémoire et l'oubli à une certaine qualité de l'âme ( $\psi v \chi \eta ́)$, pour le premier, ou du cerveau ( $\dot{\varepsilon} \gamma \kappa \varepsilon \phi \alpha ́ \lambda ం \varsigma)$, pour le second ; voir Hippocrate (Joly 1967), Du régime, I, 35, 1: l'âme composée du feu le plus humide et de l'eau la plus sèche « est la plus intelligente et a la

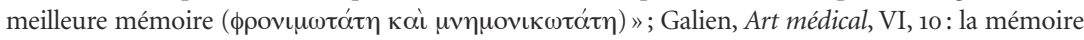
est la marque d'une substance stable ( $\mu$ ovíuos oúoí $\alpha$ ) du cerveau; l'oubli, d'une substance fluide

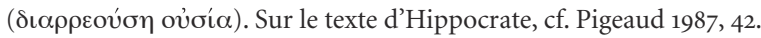

42. Voir Debru 2002, 78: «Chez les Romains, par exemple, depuis Celse et Pline jusqu'aux auteurs de l'époque tardive, on s'intéresse peu à l'anatomie et à la physiologie du corps normal. La littérature encyclopédique ou médicale les sous-entend plutôt qu'elle ne les détaille, préférant s'attacher aux maux et à leur traitement. Cela ne signifie pas que cette médecine ne s'intéresse pas au corps. Mais le corps n'y existe que lorsqu'il est médicalisé, c'est-à-dire soumis à différents processus qui le reconfigurent. Ce n'est pas le corps lui-même, mais son altération qui est l'objet de la médecine. »

43. Cette expression est empruntée à Simondon 1982, 135 sq. L'auteur désigne sous ce nom des substances qui «sont à la fois un mal et un bien, poison et remède » (ibid., 135), parce qu'elles donnent un oubli qui fait plaisir, momentanément ou durablement, mais qu'elles sont en même temps dangereuses. Parmi ces nourritures, il y a le népenthès qu'Hélène jette dans le vin pour ôter aux convives de Sparte les souvenirs des maux de Troie (Odyssée, IV, 220 sq.), le lotus, qui donne l'oubli du retour et qui apparaît dans l'épisode des Lotophages (Odyssée, IX, 94 sq.). La drogue de Circé ôte elle aussi tout souvenir de la patrie (Odyssée, X, 236 sq.). Quant à Calypso (Odyssée, V, 197 sq.), elle offre à Ulysse, pour le retenir auprès d'elle, «l'éternel oubli de la vie divine», sous la forme du nectar et de l'ambroisie (Simondon 1982, 139). À Rome, le lotus est toujours cité comme une nourriture d'oubli : voir Pline l'Ancien, Histoire naturelle, XIII, 105. Les Romains attribuent aussi le pouvoir de donner l'oubli au pavot; voir Ovide, Fastes, IV, 533 : «On raconte que, pendant qu'il cueille le pavot, il l'a goûté dans 
qui provoquent l'oubli. Ces «nourritures » sont ambivalentes: c'est le contexte qui détermine si leur usage est moralement condamnable, ou au contraire licite et même souhaitable. À Rome, la nourriture d'oubli par excellence est le vin, souvent associé au sommeil. Ainsi, dans les textes comiques, mais aussi dans l'invective politique, sont mis en relation le fait de boire (de façon inconsidérée, honteuse) et celui de perdre la mémoire. Soit cette conduite est le fait d'êtres qui ont à la fois par leur nature et leur position sociale tendance à l'oubli, c'est-à-dire des esclaves et des affranchis ${ }^{44}$, soit elle est attribuée à des membres de la nobilitas, voire des Princes, qui sont débauchés et qui s'adonnent au vin et au sommeil ${ }^{45}$. Le vin comme le sommeil sont contraires à l'industria et au pudor, quand ils sont installés au cour d'un espace (la vie politique) où ils ne peuvent être que perturbateurs.

En revanche, l'oubli apporté par le vin a une valeur positive lorsqu'il intervient dans le cadre du banquet, qui, lui, relève de l'otium ${ }^{46}$. Cet oubli est nécessaire, car il permet de quitter l'espace du negotium. Dans le même contexte, l'amour joue un rôle analogue au vin et provoque l'oubli ${ }^{47}$. Ainsi, Horace ne peut plus écrire de poésie, car il est amoureux de l'affranchie Phryné; il s'adresse à Mécène en ces termes :

son palais oublieux» (oblito palato) (l'adjectif a une valeur résultative). Le pavot est aussi associé au sommeil - qui est une figure de l'oubli - comme l'attestent le discours médical aussi bien que la poésie; voir par exemple Celse, De medicina, V, 254: le pavot sauvage entre dans la composition d'un remède qui fait dormir ; Virgile, Énéide, IV, 486 : le pavot est qualifié de "soporiferum " (qui apporte le sommeil).

44. Pour les esclaves, voir Plaute, Trinummus (1013-1014 et 1017-1018) : l'esclave Stasimus se blâme luimême pour avoir bu et pour avoir, du coup, « oublié sa mémoire» ( Memoria $<m>$ esse oblitum?»); Amphitryon, 630-631 (Sosie à Amphitryon : «J'ai de la mémoire et du zèle pour que ce que tu ordonnes soit exécuté. Je n'ai pas avalé tes ordres dans un verre de vin. » [Et memor sum et diligens, ut quae imperes compareant. / Non ego cum uino simitu ebibi imperium tuum]); la même idée apparaît dans le Persa, 170. Voir aussi Columelle, De re rustica, XI, 1, 13: l'intendant d'une ferme doit faire un usage modéré du vin, du sommeil et des plaisirs de l'amour, qui sont les ennemis de l'attention dans le travail (diligentia), du souci de la tâche (cura officii), de la mémoire (memoria).

45. Voir Cicéron, De haruspicum responsis, 55, à propos de Clodius : «Sans doute est-il plein de vin, de débauche, de sommeil, plein de la plus démente et de la plus folle irréflexion » (Est quidem ille plenus uini, stupri, somni, plenusque inconsiderantissimae ac dementissimae temeritatis). Si Cicéron ne parle pas explicitement d'oubli, tous les termes qu'il emploie lui sont associés et s'opposent à la mémoire, à la prudence. Voir aussi Tacite, Histoires, III, 55 sq., à propos de Vitellius, et Histoire auguste, Les deux Maximins, 17, 5 : apprenant que Gordien est Empereur, avec l'approbation du Sénat, et que ces deux autorités réclament sa mort, Maximin est pris de folie furieuse : « [...] comme il ne pouvait contenir sa fureur, pour oublier cette pensée, le premier jour, il se noya de vin, dit-on, jusqu’à ne plus savoir ensuite ce qui s'était passé» (Sed cum furorem suum tenere non posset, ut obliuionem cogitationis acciperet, uino se primo die obruisse dicitur, eo usque ut, quid actum esset, ignoraret).

46. Horace, Odes, II, 7, 21. Le Massique est «obliuiosus» : il donne l'oubli.

47. Voir par exemple Térence, Eunuque, 296 sq. 
Pourquoi une paresse amollissante a répandu un tel oubli au plus profond de mes sens, comme si j'avais aspiré, d'une gorge desséchée, des coupes versant avec elles un sommeil léthéen, tu me tues, loyal Mécène, à me le demander sans cesse ${ }^{48}$.

Ces quelques vers recensent toutes les nourritures et les conduites d'oubli traditionnelles: la paresse (inertia), le vin et le Léthé, qui est associé au sommeil ${ }^{49}$; l'amour, qui est la cause de tout cela, apparaît dans la suite du poème. Il est possible, en outre, que, dans cette Épode, l'image de la diffusion de l'oubli dans tout le corps soit une allusion au poison; en effet, la perte de la mémoire paraît être un des symptômes caractéristiques de l'empoisonnement ${ }^{50}$.

Ces différents exemples confirment à quel point la mémoire est constitutive de l'individu et de son intégrité, sur le plan biologique comme sur le plan culturel.

Cet aspect est sensible dans un texte de fiction comme les Métamorphoses d'Ovide. En effet, s'il n'y est nullement question de mémoire du corps - d'un corps qui garderait la mémoire de ce qu'il a été ou au contraire qui l'oublierait -, il apparaît que c'est la mémoire qui assure une permanence de l'identité, au-delà du corps et de ses métamorphoses. Ainsi, les objets et les êtres qui ont subi une transformation se souviennent (ils sont memores) de ce qu'ils ont été, de leur passé, du malheur qui a provoqué la transformation de leurs corps, et leur comportement est conditionné par ce souvenir ${ }^{51}$. La mémoire fait le lien entre passé, présent et avenir; elle organise l'action ${ }^{52}$.

48. Horace, Épodes, 14, 1-5: «Mollis inertia cur tantam diffuderit imis / obliuionem sensibus, / pocula Lethaeos ut si ducentia somnos arente fauce traxerim, / candide Maecenas, occidis saepe rogando [...].»

49. Voir Simondon 1982, 131 sq. sur la mythologie grecque du Léthé. À Rome, il n’y a pas de mythologie du Léthé, du sommeil ou de certaines nourritures, mais le sommeil et le Léthé sont des figures poétiques de l'oubli. De plus, le sommeil et certains comportements (inertia, mollitia, etc.) associés à l'ingestion de certaines substances (vin, poisons ou remèdes) se rattachent à la catégorie culturelle de l'otium, qui s'oppose à celle du negotium et à ses valeurs (l'industria, le labor...).

50. Voir Juvénal (de Labriolle, Villeneuve \& Gérard 1983), VI, 610-614: «Parmi ces charlatans, l’un apporte des chants magiques, l'autre vend des philtres thessaliens grâce auxquels la femme abrutira son mari et le fessera à coups de savate. Si tu perds le sens, là en est la cause; c'est de là que te viennent des nuages qui t'obscurcissent l'âme; cet oubli complet de tes actions les plus récentes (quod desipis, inde est, / inde animi caligo et magna obliuio rerum / quas modo gessisti).»

51. Voir Ovide, Métamorphoses, XIV, 558-565: les navires d’Énée brûlés par Turnus ont été transformés par Cybèle en naïades de la mer ; celles-ci n'ont pas oublié (non oblitae) les dangers de la navigation et se souviennent (memores) des malheurs des Troyens ; IV, 603 : Cadmus et Harmonie transformés en serpents se souviennent de ce qu'ils ont été autrefois ( $Q$ Quidque prius fuerint placidi meminere dracones»); voir aussi VIII, 259 (Perdix). Inversement, Callisto, transformée en ourse, oublie parfois ce qu'elle est devenue (Ovide, Métamorphoses, II, 493: «Saepe feris latuit uisis, oblita quid esset »).

52. Sur cette idée, voir Simondon 1982, 11 et 306-309 (p. 306: «Un préjugé répandu depuis l'antiquité jusqu'aux théoriciens modernes de la mémoire donne un privilège au passé enregistré ou à l'habitude acquise ou à la fixation et à l'évocation du souvenir, sans accorder d'attention à une forme de mémoire liée à ce que nous appelons le projet d'action. »). 


\section{La mémoire, l'oreille, l'estomac et autres parties du corps}

Si le fonctionnement de la mémoire est lié à l'état général du corps, la memoria est aussi plus spécifiquement associée à certaines parties du corpus, en particulier l'oreille et l'estomac. Loin de faire apparaitre un corps morcelé ou, au contraire, de dessiner une sorte de physiologie unique du corps, ce lien entre mémoire et parties du corps montre de nouveau à quel point la mémoire est pensée à Rome comme une fonction, qui organise des comportements dans un contexte donné.

En ce qui concerne l'oreille ${ }^{53}$, tout d'abord, un passage étonnant de Pline l'Ancien explique que «le lobe de l'oreille est le lieu de la mémoire »; Pline explique aussitôt cette localisation par une pratique, en disant que c'est «l'endroit que nous touchons quand nous demandons un témoignage à quelqu'un ${ }^{54}$. L'oreille et la mémoire sont ici mises en relation par un geste, qui sert à demander à quelqu'un de témoigner de ce qu'il a vu et entendu. Cet usage est attesté par d'autres textes ${ }^{55}$. De façon générale, toucher ou tirer l'oreille de quelqu'un consiste à lui rappeler quelque chose ( $\mathrm{admo-}$ nere), à solliciter sa mémoire ${ }^{56}$.

D'autres documents, textes littéraires ${ }^{57}$ mais aussi inscriptions ${ }^{58}$ et représentations figurées ${ }^{59}$, montrent l'importance de l'oreille des dieux dans le culte: leur parler à l'oreille, remercier leurs « oreilles» dans une dédicace, c'est en même temps s'adresser à leur mémoire ${ }^{60}$. Il est en effet dans la fonction des dieux de se souvenir des hommes et de leurs demandes.

Tous ces documents montrent que le lien entre l'oreille et la mémoire est bien attesté dans la culture et appartient à un savoir partagé. Ils permettent aussi de comprendre que le propos de Pline l'Ancien cité initialement ne restitue pas un savoir anatomique selon lequel la mémoire serait logée dans une partie du corps, mais qu'il rend compte d'une sorte de physiologie fonctionnelle du corps, ou, pour reprendre les termes de Marcel Mauss, de «techniques du corps ${ }^{61}$. On le comprend bien aussi

53. J'ai abordé cette question lors d'un exposé présenté dans le séminaire de Philippe Moreau à l'Université de Caen Basse-Normandie le 6 mai 2003.

54. Pline l'Ancien, Histoire naturelle, XI, 251: «Est in aure ima memoriae locus, quem tangentes antestamur $[\ldots]$..»

55. Voir Horace, Satires, I, 9, 74-77 et les commentaires ad loc. d'Acron et de Porphyrion.

56. Voir Virgile, Bucoliques, VI, 3-4 et le commentaire de Servius (voir infra, note 62).

57. Sénèque, Lettres à Lucilius, I, 10, 5 et IV, 41, 1.

58. CIL, III, $986=I L S_{3} 848 ; C I L, \mathrm{~V}, 759=I L S_{3497}$.

59. Cf. Lambrechts \& Vanden Berghe 1955. Cet article s’intéresse à deux autels: le premier, trouvé à Glanum et daté du premier siècle de notre ère, porte une dédicace faite par une auxiliaire (ministra) du culte aux « oreilles » probablement de la Bonne Déesse (Bona dea); le second vient d'Arles et il est dédié à Bona dea par un personnage analogue ; sur les deux autels sont représentées, à l'intérieur d'une couronne, deux oreilles.

60. Pour la Grèce, voir Weinreich 1912, et Versnel 1981.

61. Mauss 1936. 
en lisant un commentaire de Servius, dans lequel on retrouve la notice de Pline l'Ancien :

Les naturalistes disent que les parties du corps sont chacune consacrées à des divinités, comme l'oreille à la mémoire - de là (Bucolique, VI, 3 ) «le dieu du Cynthe me tira l'oreille et me rappela à la mémoire»-, le front au génie (genius), d'où le fait que, quand nous rendons un culte à ce dieu, nous nous touchons le front, la main droite à la fides $[\ldots]$, les genoux à la pitié, de là le fait que ceux qui font une demande les touchent ${ }^{62}$.

Ainsi, les parties du corps ne sont pas « consacrées » à une divinité au sens où elles lui appartiennent, mais elles participent à des gestes rituels qui mettent en cause une divinité (le Genius, la Fides) ou une valeur (la mémoire, la compassion) ${ }^{63}$.

D'autres parties du corps sont associées à la mémoire, en particulier dans la langue poétique. Ainsi le cœur (cor), la poitrine (pectus), la langue (lingua), la bouche (os) et la main (manus ou dextra) sont-ils qualifiés par les adjectifs memor (qui se souvient, qui a de la mémoire) ou immemor, obliuiosus et oblitus (oublieux) ${ }^{64}$. Comme l'oreille, ces organes sont définis comme doués de mémoire ou, au contraire, oublieux, lorsqu'ils interviennent dans un geste, un acte ${ }^{65}$ et dans un contexte précis; très souvent, ces formules sont à l'ablatif instrumental. Par exemple, dans le carmen 64 de Catulle, Ariane est abandonnée par un Thésée dont la poitrine est pleine

62. Servius, Énéide, III, 607: « [...] physici dicunt esse consecratas numinibus singulas corporis partes, ut aurem memoriae - hinc est (E. VI 3 ) Cynthius aurem uellit et admonuit - frontem genio, unde uenerantes deum tangimus frontem, dextram fidei, [...] genua misericordiae, unde haec tangunt rogantes."

63. Cette idée apparaît très clairement chez Pline l'Ancien lui-même, dans le passage qui précède immédiatement la notice sur l'oreille (et où l'on retrouve les éléments notés par Servius) : «Selon les préceptes populaires, il y a une sorte de respect religieux pour les genoux de l'homme. Les suppliants les touchent, tendent les mains vers eux, les adorent comme des autels [...]. À d'autres parties du corps aussi s'attache une sorte de respect religieux, par exemple la main droite: on cherche le dos de la main pour l'embrasser, on la tend pour donner sa foi. Les anciens Grecs avaient coutume de supplier en touchant le menton» (Histoire naturelle, XI, 250).

64. Certaines formules sont une périphrase du nom memoria ou du verbe memini. Voir Horace, Satires,

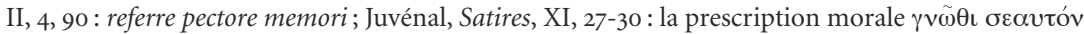
doit être «fixée dans une poitrine pleine de mémoire et doit y être méditée (figendum et memori tractandum pectore).

65. Par exemple, on rencontre une occurrence de "manus memor» et une de " os memor» chez Ovide, pour exprimer l'acte d'écrire ou de dire en rapport avec la mémoire et la commémoration: voir Pontiques, III, 4, 18: les poètes qui ont pu voir le triomphe de Tibère notent (notare) ce qu'ils ont vu (uisa) d'une main qui se souvient (memori manu); Métamorphoses, X, 204: Apollon s'accusant d'avoir causé la mort d'Hyacinthe, lui promet: «Tu seras toujours avec moi et tu resteras toujours fixé dans ma bouche qui se souviendra » (Semper eris mecum memorique haerebis in ore). Voir aussi Métamorphoses, VI, 508 (memori ore salutare) et Amores, III, 14, 48 (lingua memor). 
d'oubli $^{66}$. Dans une élégie de Properce, Tarpéia, amoureuse de Titus Tatius, laisse échapper de ses «mains oublieuses» l'urne avec laquelle elle allait puiser de l'eau pour le culte de Vesta ${ }^{67}$. On peut penser que ces images ne sont pas de simples figures de style (des métonymies et des hypallages). En effet, elles permettent de repérer des situations (la mémoire des promesses, l'échange de bienfaits, l'oubli des obligations cultuelles au profit de l'amour) dans lesquelles la mémoire et l'oubli jouent un rôle déterminant. En outre, si ces expressions ne dessinent pas une représentation unifiée des parties du corps en tant qu'organes de la memoria et de l'obliuio, elles montrent encore une fois l'aspect fonctionnel de la mémoire et de l'oubli, qui caractérisent gestes et conduites. Comme dans le cas de l'oreille, ce qui est en jeu ici, ce n'est pas une mémoire psychologique, mais une mémoire d'action, une mémoire pragmatique, qui est actualisée par des gestes et des comportements.

Il nous reste à examiner une dernière partie du corps liée à la mémoire : l'estomac (stomachus $)^{68}$, et à étudier le rapprochement entre mémoire et digestion. Cette question renvoie à toute une représentation de l'éducation, de la mémorisation et de la remémoration ${ }^{69}$. À cet égard, le développement que Quintilien consacre à la mémoire de l'orateur (Institution oratoire, XI, 2) est particulièrement éclairant. On $\mathrm{y}$ trouve plusieurs indications qui mettent en jeu le corps. Tout d'abord, la mémoire naturelle de chacun dépend de la qualité de son tempérament $(\text { ingenium })^{70}$; en

66. Catulle, Poésies, 64, 123 (immemori pectore) et 208 (oblito pectore); voir aussi 248 (mente immemori). Dans ce poème, Thésée est en quelque sorte un héros de l'oubli, vis-à-vis d'Ariane comme d'Égée; ainsi, Égée rappelle à son fils qu'il ne doit pas oublier ce qu'il lui a prescrit (changer la voile) : «Alors, fais en sorte que mes ordres, mis en réserve dans ton cœur plein de mémoire, restent vigoureux et ne laisse pas le temps les effacer» (231-232: Tum uero facito ut memori tibi condita corde / haec uigeant mandata nec ulla obliteret aetas). Pour un exemple non poétique qui associe la poitrine et l'oubli, voir Sénèque, De beneficiis, I, $3,1:$ « [...] celui qui revient à la charge et accumule bienfait sur bienfait fait sortir la reconnaissance même d'une poitrine dure et oublieuse » (at qui instat et onerat priora sequentibus, etiam ex duro et immemori pectore gratiam extundit).

67. Properce, Élégies, IV, 4, 22: «manus oblitas excidit urna» (l'adjectif a une valeur résultative). Il y a peut-être dans ce vers un jeu sur le verbe excidere, qui signifie aussi «sortir de la mémoire (employé seul ou avec memoria).

68. Sur ce terme, voir André 1991, 132-133: stomachus, qu'on rencontre dans les textes à partir du premier siècle avant J.-C., peut désigner l'œsophage ou l'estomac.

69. Ma réflexion est ici largement tributaire des travaux d'Emmanuelle Valette-Cagnac 1993, 138 sq. et 2002, en particulier 299-303.

70. Quintilien, Institution oratoire, XI, 2, 42: «[...] ceux qui ont un tempérament un peu plus lent que les autres n'ont pas une bonne mémoire du passé récent» ([...] minime fidelem esse paulo tardioribus ingeniis recentem memoriam). L'absence de mémoire, ou plutôt d'un certain type de mémoire, est ici associée à la lenteur, comme dans le Cato Maior, 21 (voir supra note 33). Dans son traité sur la mémoire (De memoria et reminiscentia, 449b, 6-9), Aristote établit une différence entre ceux qui sont lents et ceux qui sont rapides : «[...] ce ne sont pas les mêmes personnes qui ont de la mémoire et qui se souviennent par réminiscence; mais le plus souvent, ce sont les gens lents qui ont plus de mémoire, et ceux qui se souviennent le plus facilement sont ceux qui sont vifs et intelligents » (oú 
outre, la mémoire est particulièrement lente (tardior) ou solide (firmior) selon la constitution $(\text { habitus })^{71}$; enfin, pour bien se souvenir, il faut veiller à l'hygiène du corps et de l'âme. Ainsi, il explique:

Il y a quelque chose de commun à l'acte d'apprendre par cour et à celui d'écrire: ce qui est le plus utile dans les deux cas, c'est une bonne santé, une nourriture bien digérée, une âme libre de toute autre pensée ${ }^{72}$.

L'orateur qui prépare ses discours doit donc avoir le corps et l'âme dégagés, libres de tout processus de digestion ou de réflexion. En fait, le conseil de Quintilien relève de toute une représentation du corps méditant, lisant, écrivant ${ }^{73}$.

L'orateur doit encore exercer sa mémoire et la nourrir, comme un athlète le ferait pour son corps : à propos des exercices qui sont pratiqués par l'enfant, futur orateur, et qui consistent pour lui à mémoriser ce qu'il a lu, en particulier des passages choisis de textes poétiques, Quintilien explique que «c'est surtout par l'exercice qu'on affermit et qu'on nourrit» la mémoire ${ }^{74}$. Cet aspect est solidaire de tout un système de pensée dans lequel l'enseignement consiste à nourrir, comme l'indique le double sens du verbe latin alere: nourrir et élever, amener à l'âge adulte ${ }^{75}$. De plus, la mémoire s'exerce, comme le corps ; on a vu que Caton recommandait cet entraînement aux senes.

En outre, la lecture d'auteurs, qui est un des éléments essentiels de la formation de l'orateur, est conçue comme une ingestion, une mastication et une digestion. Ainsi, Quintilien, dans un chapitre consacré aux lectures utiles à l'orateur $(\mathrm{X}, 1)$, donne le conseil suivant :

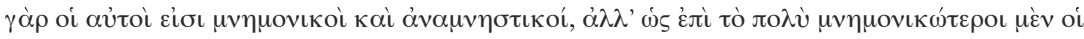

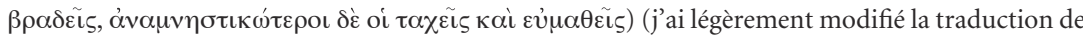
R. Mugnier, 1965). Cette remarque repose sur la distinction entre mémoire et remémoration ou réminiscence, qui, encore une fois, n'est pas pratiquée dans les textes latins.

71. Ibid., $4:$ «[...] la mémoire est particulièrement lente ou solide selon notre constitution » (habitu tardiorem firmioremque memoriam fieri). Voir aussi XI, 2, 44.

72. Ibid., 35: «Illud ediscendo scribendoque commune est, utrique plurimum conferre bonam ualetudinem, digestum cibum, animum cogitationibus aliis liberum. »

73. Voir aussi ibid., 3, 19: pour prendre soin de sa voix, l'orateur doit veiller à la fermeté de son corps (firmitas corporis) et, à cette fin, avoir une digestion facile (facilis ciborum digestio) et respecter en tout la frugalité (frugalitas). Voir encore X, 3, 26 et les analyses d'E. Valette-Cagnac (1993, 154 et 2002, 302-303), qui montrent qu'il existait une véritable diététique de la lecture à Rome et que celle-ci rentrait dans une conception de l'ingestion et de l'assimilation du savoir.

74. Quintilien, Institution oratoire, I, 1,36: « [...] et ea praecipue firmatur atque alitur exercitatione. » La notion de firmitas (fermeté, solidité, durée aussi) est essentielle pour définir la mémoire: voir Rhétorique à Hérennius, I, 3 ; Cicéron, De inuentione, I, 9 ; Quintilien, Institution oratoire, XI, 2, 2 et 4 (cité supra note 71) et 35; Diomède, in H. Keil, Grammatici latini, I, Hildesheim, G. Olms, 1961, 419.

75. Voir Dupont 2002, 115 et 136; le nourrissage est biologique, mais aussi culturel (136: «Ce qui est en jeu est la transmission du corpus et de l'animus du père, la formation du corps culturel de l'enfant»). 


\section{Corps et MÉmoIre À Rome}

On peut revenir [sur le texte] à plusieurs reprises, soit qu'on ait des doutes, soit qu'on veuille le fixer profondément dans la mémoire. Revenons-y et reprenons-le, et, de même que les aliments sont mâchés et presque liquéfiés quand nous les avalons, afin de faciliter la digestion, ce que nous lisons ne doit pas non plus être transmis tout cru à la mémoire et à l'imitation, mais par de fréquentes reprises, doit être amolli et, pour ainsi dire, absorbé ${ }^{76}$.

Ici, la digestion est conçue comme une coction (coctio ou concoctio) des aliments, qui sont crus, conformément à la théorie hippocratique reprise dans les traités romains ${ }^{77}$. C'est cette transformation opérée par la digestion qui permet aux aliments d'être assimilés par le corps et de faire partie de lui ${ }^{78}$. Le texte, quant à lui, doit être mâché comme un aliment (c'est-à-dire lu et relu) pour être mieux digéré par le corps ${ }^{79}$. La mémoire ne doit pas jouer le rôle d'un estomac qui cuit les aliments ${ }^{80}$, car les textes-aliments doivent lui parvenir digérés (c'est-à-dire cuits), mais elle a un rôle de conservation. Le danger est qu'y reste fixée une lecture " crue », qui n'aura pas vraiment été assimilée et qui sera reproduite telle quelle. C'est ce que fait comprendre le terme imitatio, ici associé à memoria. L'imitatio peut être définie comme la faculté de produire des énoncés en se souvenant d'autres énoncés, qui jouent le rôle de modèles, mais elle suppose que l'on se soit approprié ces modèles. L'imitation n'est nullement une reproduction à l'identique.

Dans une Lettre à Lucilius, Sénèque a lui aussi recours à la comparaison entre assimilation du savoir et digestion; si le terme d'imitation n'y figure pas, l'idée, elle, est bien présente. Voici en effet ce que Sénèque conseille à Lucilius :

Les aliments ingérés, tant qu'ils se conservent tels quels et qu'ils nagent à l'état solide dans l'estomac, sont une charge. Mais quand ils se sont transformés, alors, et alors seulement, ils se changent en forces et en sang. Procédons de même pour les nourritures de l'intelligence: faisons en sorte que tout ce que nous avons absorbé ne demeure

76. Quintilien, Institution oratoire, X, 1, 19: «[... ] repetere saepius licet, siue dubites siue memoriae penitus adfigere uelis. Repetamus autem et tractemus, et, ut cibos mansos ac prope liquefactos demittimus, quo facilius digerantur, ita lectio non cruda, sed multa iteratione mollita et uelut confecta memoriae imitationique tradatur.»

77. Voir Celse, De medicina, préface 38. Sur les théories antiques de la digestion, voir Pigeaud 1981, 180186 et 198.

78. Voir Galien, Des facultés naturelles, II, 4, 89: «La coction est une altération et une transformation de l'aliment en la qualité propre du corps nourri» (traduction Pigeaud 1981, 182, note 184).

79. Valette-Cagnac 1993, 139: «La lecture n'est pas conçue comme un processus de digestion complet; elle correspond à la phase de mâchage, où la salive produit une première forme de cuisson et de digestion. [...] elle permet la transformation en sucus, substance homogène, d'une variété d'aliments hétérogènes. "

80. Voir Galien, Art médical, XVII, 3 sur les marques disctinctives et les différents types d'estomac $(\gamma \alpha \sigma \tau \eta ́ \rho)$ : lors de la digestion, un estomac qui est «plus chaud par nature » cuit les aliments. 
pas intact en nous, de peur que cela ne nous reste étranger. Digérons ces nourritures : sinon elles passeront dans notre mémoire, non dans notre intelligence ${ }^{81}$.

Là aussi, le travail de digestion doit être fait juste après l'ingestion afin que la mémoire ne stocke pas une nourriture crue, non assimilée. C'est en ce sens que la memoria est ici distinguée de l'ingenium, qui permet de s'approprier et de réutiliser un savoir.

Bien après Sénèque et Quintilien, la comparaison alimentaire et digestive est utilisée à propos de la mémoire par Augustin. Cependant, elle n'a pas le même sens et elle permet, de ce fait, de comprendre la particularité des représentations antérieures. Voici ce qu'écrit Augustin:

Sans doute la mémoire est-elle comme l'estomac [uenter] de l'âme, et la joie ou la tristesse comme une nourriture douce ou amère; lorsque ces sentiments sont confiés à la mémoire, après être passés, en quelque sorte, dans cet estomac, ils peuvent s'y enfermer, mais ils n'ont plus de goût.

Il ajoute un peu plus loin :

Peut-être que, comme la rumination ramène de l'estomac la nourriture, la remémoration ramène de la mémoire des sentiments ${ }^{82}$.

Ici, la mémoire est clairement comparée à un estomac. Mais, si la mémoire a, là aussi, un rôle de conservation (recondi), elle dénature ce qu'elle conserve (sapere non possunt), les aliments concernés sont des sentiments (la joie, la tristesse), non des connaissances, et, surtout, il n'est pas question ici de digérer des aliments pour mieux les réutiliser, alors que la notion d'assimilation du savoir et celle de l'imitation sont fondamentales chez Sénèque et Quintilien. En outre, cette comparaison ne sert pas à valoriser la mémoire des sentiments, sans doute parce que, chez le chrétien Augustin, le corps a une autre place et que c'est l'âme qui prévaut.

81. Sénèque, Lettres à Lucilius, XI, 84, 6-7: «[...] alimenta, quae accepimus, quamdiu in sua qualitate perdurant et solida innatant stomacho, onera sunt; at cum ex eo, quod erant, mutata sunt, tum demum in uires et in sanguinem transeunt. Idem in his, quibus aluntur ingenia, praestemus, ut quaecumque hausimus, non patiamur integra esse, ne aliena sint. Concoquamus illa: alioquin in memoriam ibunt, non in ingenium. » Valette-Cagnac 1993, 171, remarque que cette représentation de la digestion est parfaitement conforme au discours médical de l'époque.

82. Augustin, Confessions, $\mathrm{X}, 14,21$ : «Nimirum ergo memoria quasi uenter est animi, laetitia uero atque tristitia quasi cibus dulcis et amarus: cum memoriae conmendantur, quasi traiecta in uentrem recondi illic possunt, sapere non possunt. » et 22 : "Forte ergo sicut de uentre cibus ruminando, sic ista de memoria recordando proferuntur. » Le terme uenter (estomac) apparaît avec Scribonius Largus au premier siècle après J.-C.; il est utilisé par Pline l'Ancien et jusqu’à la basse latinité (André 1991, 133). 
Citons enfin un dernier extrait de l'Institution oratoire, assez difficile à comprendre, qui associe l'image de la fermeté de la mémoire à celle de la digestion :

Il est étonnant, et difficile à expliquer, à quel point un intervalle d'une nuit apporte de la fermeté à la mémoire, soit qu'il la repose de son travail, dont la fatigue était pour elle un obstacle, soit que la remémoration [recordatio], qui est la partie la plus ferme de celle-ci, soit mûre et digérée ${ }^{83}$.

Ici, la réminiscence (recordatio) apparaît comme l'élément actif de la mémoire. Or, par un effet analogique, c'est parce qu'elle est la plus ferme (firmissima) qu'elle peut donner de la fermeté (firmitas) à la mémoire. De plus, cette partie de la mémoire elle-même est digérée. Peut-être faut-il mettre en relation la métaphore de la mémoiredigestion ainsi formulée et rapportée à la recordatio avec la représentation des aliments. En effet, ils mûrissent, ils sont digérés et, d'après Celse, ils peuvent être «fermes » et, de ce fait, plus ou moins faciles à digérer ${ }^{84}$. Dans l'extrait de Quintilien, tout se passe comme si la mémoire devait, au terme du processus de l'ingestion et de la digestion du texte-aliment, avoir la même qualité que les aliments qu'elle ingère, sans déperdition. Cependant, il n'est ni certain ni nécessaire que cette métaphore d'une «remémoration digérée» soit parfaitement cohérente avec les autres textes de Quintilien et avec celui de Sénèque que nous avons cités et participe d'une représentation unifiée de la mémoire.

Passer en revue les parties du corps qui sont associées à la mémoire nous a donc permis de comprendre plusieurs points importants. Les gestes accomplis dans le cadre de rituels religieux ou sociaux (la demande de témoignage, par exemple) font voir qu'il existe une mémoire d'action, repérable en particulier dans les relations d'échange. En outre, en examinant les textes de Sénèque et de Quintilien qui définissent l'assimilation des connaissances comme une digestion, on voit que la mémoire permet de conserver le savoir et que ce rôle entre dans une conception plus générale de la

83. Quintilien, Institution oratoire, XI, 2, 43: «Mirum dictu est nec in promptu ratio quantum nox interposita adferat firmitatis, siue requiescit labor ille, cuius sibi ipsa fatigatio obstabat, siue maturatur atque concoquitur, quae firmissima eius pars est, recordatio [...].» J. Cousin, traducteur pour la CUF, et H.E. Butler, dans l'édition Loeb, traduisent «firmus» par «important», mais firmus n'a jamais ce sens.

84. Celse (De medicina, II, 18, 1 sq.) distingue trois classes d'aliments : la première rassemble les plus nourrissants (genus ualentissimum "in quo plurimum alimenti est»); la seconde est intermédiaire et la troisième comprend les nourritures les plus «faibles» (imbecillissima materia). À l'intérieur de ces classes, certains aliments peuvent être plus nourrissants que d'autres; ces qualités sont définies par plusieurs adjectifs, dont l'adjectif firmus, qui paraît mis sur le même plan que grauis ou ualens. Enfin, plus un aliment est nourrissant, plus il est difficile à digérer, mais, s'il est digéré, il est plus nourrissant (13: «Fere uero sequitur, ut, quo ualentior quaeque materia est, eo minus facile concoquatur, sed, si concocta est, plus alat.»). 
production ou de l'imitation d'énoncés, dans laquelle corpus, animus, ingenium sont indissociablement liés.

\section{Conclusion}

Au terme de cette étude, on peut dire que la mémoire est un bon exemple de l'absence de séparation systématique et tranchée entre corpus, d'une part, et animus ou mens ou ingenium, d'autre part, absence de séparation qui caractérise la société et les discours à Rome, pour la période que nous avons envisagée. Dans les différentes représentations que nous avons rencontrées, la mémoire se constitue en même temps que l'homme et avec lui : grâce à la nourriture et à l'entraînement, elle devient ferme; lorsque l'homme est vieux, elle s'affaiblit ou s'enfuit. Elle est donc solidaire à la fois de son corpus et de son animus; elle est un des éléments qui composent et font vivre son corps culturel.

D’autre part, dans les textes examinés, il y a des liens multiples entre le corps et la mémoire, qu'il s'agisse de parties du corps ou bien du corps tout entier. Ces liens, établis et vivifiés par la langue et par des pratiques (celle qui consiste à toucher l'oreille, par exemple), indiquent que la mémoire comme l'oubli, à Rome, caractérisent les hommes comme êtres sociaux, dans leurs relations avec les autres et dans leurs conduites. En cela, les images et les comparaisons qui décrivent la mémoire, son fonctionnement et ses implications, permettent bien d'avoir accès à un corps «réel».

Catherine BAROIN

Université de Rouen

\section{Références bibliographiques}

\section{Auteurs anciens (textes principaux)}

Aristote, De memoria et reminiscentia, in Petits traités d'histoire naturelle, R. Mugnier (éd. et trad.) $\left(1965,1953^{1}\right)$, Paris, Les Belles Lettres (CUF).

Augustin, Confessions, IX-XIII, P. de Labriolle (éd. et trad.) (1926), Paris, Les Belles Lettres (CUF).

Catulle, Poésies, G. Lafaye (éd. et trad.) (1923), Paris, Les Belles Lettres (CUF).

Celse, De medicina, I-II, G. Serbat (éd. et trad.) (1995), Paris, Les Belles Lettres (CUF).

Celse, De medicina, W. G. Spencer (trad.) (1935-1938), Londres, Loeb.

Cicéron, Cato Maior, P. Wuilleumier (éd. et trad.) (1940), Paris, Les Belles Lettres (CUF). 


\section{Corps et mémoire À Rome}

Cicéron, De inuentione, G. Achard (éd. et trad.) (1994), Paris, Les Belles Lettres (CUF).

Cicéron, De haruspicum responsis, P. Wuilleumier, A.-M. Tupet (éd. et trad.) (1966), Paris, Les Belles Lettres (CUF).

Cicéron, De legibus, G. de Plinval (éd. et trad.) (1959), Paris, Les Belles Lettres (CUF).

Cicéron, Tusculanes, I-II, G. Fohlen (éd.), J. Humbert (trad.) (1968, 1931¹), Paris, Les Belles Lettres (CUF).

Columelle, Res rustica, X-XII, E.S. Forster, E.H. Heffner (éd. et trad.) (1968, 1955'), Londres, Loeb.

Galien, Art médical, V. Boudon (éd. et trad.) (2000), Paris, Les Belles Lettres (CUF). Hippocrate, Du régime, R. Joly (éd. et trad.) (1967), Paris, Les Belles Lettres (CUF).

Histoire Auguste, A. Chastagnol (éd. et trad.) (1994), Paris, R. Laffont (Bouquins). Horace, Odes et Épodes, F. Villeneuve (éd. et trad.) (1946, 1929¹), Paris, Les Belles Lettres (CUF). Horace, Satires, F. Villeneuve (éd. et trad.) (1969, 1932²), Paris, Les Belles Lettres (CUF). Isidore de SÉville, Étymologies, W.M. Lindsay (éd.), vol. 1 (1989, 1911¹), Oxford (OCT). JuvénAL, Satires, P. de Labriolle, F. Villeneuve (éd. et trad.) (1983), Paris, Les Belles Lettres (CUF) $\left(12^{\mathrm{e}}\right.$ tirage revu et corrigé par J. Gérard; $\left.1921^{1}\right)$.

Ovide, Fastes, IV-VI, R. Schilling (éd. et trad.) (1993), Paris, Les Belles Lettres (CUF). Ovide, Métamorphoses, G. Lafaye (éd. et trad.) (1925-1930), Paris, Les Belles Lettres (CUF). Ovide, Pontiques, J. André (éd. et trad.) (1977), Paris, Les Belles Lettres (CUF).

Plaute, Comédies, A. Ernout (éd. et trad.) (1932-1947), Paris, Les Belles Lettres (CUF).

Pline L'Ancien, Histoire naturelle, VII, R. Schilling (éd. et trad.) (1977), Paris, Les Belles Lettres (CUF).

Pline l’Ancien, Histoire naturelle, XI, A. Ernout, R. Pépin (éd. et trad.) (1947), Paris, Les Belles Lettres (CUF).

Quintilien, Institution oratoire, I, J. Cousin (éd. et trad.) (1975), Paris, Les Belles Lettres (CUF). Quintilien, Institution oratoire, X-XI, J. Cousin (éd. et trad.) (1979), Paris, Les Belles Lettres (CUF).

Quintilien, Institution oratoire, X-XII, H.E. Butler (trad.) (1979, 1922 $\left.{ }^{1}\right)$, Londres, Loeb.

SÉnèQue le RhÉTeur, Controverses, H. Bornecque (éd.) (1932), Paris, Garnier.

SÉNÈQue, De beneficiis, I-IV, F. Préchac (éd. et trad.) (1926), Paris, Les Belles Lettres (CUF).

SÉnèque, Lettres à Lucilius, VIII-XIII, F. Préchac (éd.), H. Noblot (trad.) (1958), Paris, Les Belles Lettres (CUF).

Servius, In Vergilii Carmina Commentariorum Editio Harvardiana, E. K. Rand, H. T. Smith (éd.) (1946-1965), Lancaster - Oxford.

VArron, De lingua Latina, P. Flobert (éd. et trad.) (1985), Paris, Les Belles Lettres (CUF). Virgile, Énéide, J. Perret (éd. et trad.) (1977-1980), Paris, Les Belles Lettres (CUF). 


\section{STATUT ET IMAgE DU CORPS}

\section{Articles et ouvrages}

André J. (1991), Le Vocabulaire latin de l'anatomie, Paris, Les Belles Lettres.

BARoin C. (1998), Mémoires romaines, thèse de doctorat, sous la direction de F. Dupont, EPHE ( $\mathrm{V}^{\mathrm{e}}$ section).

Chantraine P. (1968-1975), Dictionnaire étymologique de la langue grecque, Paris, Klincksieck.

Debru A. (2002), "Altération et démembrement : corps romains médicalisés », in Moreau (éd.) 2002, p. 77-88.

Dupont F. (2002), «Le lait du père romain », in Moreau (éd.) 2002, p. 115-137.

Ernout A., Meillet A. $\left(1967^{4}\right)$, Dictionnaire étymologique de la langue latine, Paris, Klincksieck.

Kajanto I. (1982), The Latin cognomina, Rome, G. Bretschneider (Helsinki, 1965').

Lambrechts P., Vanden Berghe L. (1955), «La divinité-oreille dans les religions antiques », $B I B R$, XXIX, p. 177-198.

Le Breton D. (1985), Corps et Sociétés. Essai de sociologie et d'anthropologie du corps, Paris, Librairie des Méridiens.

Mauss M. (1936), «Les techniques du corps », Journal de psychologie, repris dans Sociologie et Anthropologie, Paris, PUF, 1950, p. 363-386.

Moreau P. (éd.) (2002), Corps romains, Grenoble, J. Millon.

Pigeaud J. (1981), La Maladie de l'âme. Étude sur la relation de l'âme et du corps dans la tradition médico-philosophique antique, Paris, Les Belles Lettres.

Pigeaud J. (1987), Folie et Cures de la folie chez les médecins de l'Antiquité gréco-romaine. La manie, Paris, Les Belles Lettres.

Simondon M. (1982), La Mémoire et l'Oubli dans la pensée grecque jusqu'à la fin du ve siècle avant J.-C., Paris, Les Belles Lettres.

Valette-Cagnac E. (1993), Anthropologie de la lecture dans la Rome antique, thèse de doctorat, sous la direction de F. Dupont, EPHE ( $V^{\mathrm{e}}$ section).

Valette-Cagnac E. (2002), « Corps de lecteurs », in Moreau (éd.) 2002, p. 289-312.

Versnel H.S. (1981), "Religious mentality in ancient prayer ", in Faith, hope and worship. Aspects of religious mentality in the ancient world, H.S. Versnel (éd.), Leyde, Brill, 1981, p. 1-64.

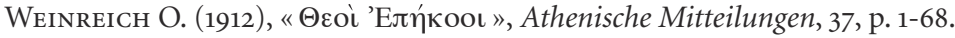

